PROCEEDINGS OF THE

AMERICAN MATHEMATICAL SOCIETY

Volume 135, Number 10, October 2007, Pages 3317-3327

S 0002-9939(07)08929-0

Article electronically published on May 17, 2007

\title{
STABILITY OF PERIODIC SOLUTIONS FOR LIPSCHITZ SYSTEMS OBTAINED VIA THE AVERAGING METHOD
}

\author{
ADRIANA BUICĂ AND ARIS DANIILIDIS \\ (Communicated by Carmen C. Chicone)
}

\begin{abstract}
Existence and asymptotic stability of the periodic solutions of the Lipschitz system $x^{\prime}(t)=\varepsilon F(t, x, \varepsilon)$ is hereby studied via the averaging method. The traditional $C^{1}$ dependence of $F(s, \cdot, \varepsilon)$ on $z$ is relaxed to the mere strict differentiability of $F(s, \cdot, 0)$ at $z=z_{0}$ for $\varepsilon=0$, giving room to potential applications for structured nonsmooth systems.
\end{abstract}

\section{INTRODUCTION}

We consider the following differential system:

$$
x^{\prime}(t)=\varepsilon F(t, x, \varepsilon)
$$

where $F: \mathbb{R} \times \Omega \times[0,1] \rightarrow \mathbb{R}^{n}$ is a continuous function, T-periodic in the first variable and locally Lipschitz with respect to $x$ (uniformly with respect to the other two variables). The set $\Omega$ is an open connected subset of $\mathbb{R}^{n}$. In this paper we are interested in the problem of existence and stability of $T$-periodic solutions of system (1.1). For sufficiently small values of the parameter $\varepsilon$, the system (1.1) is usually studied via the averaging method; see for example [13, 7, 6, 14, 8. According to this method, we consider the function $f: \Omega \rightarrow \mathbb{R}^{n}$ defined by

$$
f(z)=\int_{0}^{T} F(s, z, 0) d s .
$$

Roughly speaking, the existence of a "non-degenerate" zero $z_{0} \in f^{-1}(0)$ of the function $f$ ensures, for all small values of $\varepsilon>0$, the existence of a $T$-periodic solution of the system (1.1). A typical assumption is that $z \mapsto F(s, z, \varepsilon)$ is of class $C^{k}(k \geq 1)$ (see for instance [13, 7, 6]). In this case, the aforementioned nondegenerancy condition simply means that the determinant of the Jacobian of $f$ at $z_{0}$ does not vanish, that is, $\operatorname{det} J f\left(z_{0}\right) \neq 0$. Moreover, when $J F$ has a continuous dependence on the parameter $\varepsilon$, a study of the asymptotic stability of the periodic solutions (depending on the eigenvalues of the Jacobian matrix of $f$ at $z_{0}$ ) can be

Received by the editors August 1, 2006.

2000 Mathematics Subject Classification. Primary 34C29, 34C25; Secondary 49J52.

Key words and phrases. Periodic solution, averaging method, nonsmooth Lipschitz system, Poincaré-Andronov mapping, fixed point.

The first author was supported by the "Agence universitaire de la Francophonie" (France).

The second author was supported by the MEC Grant No. MTM2005-08572-C03-03 (Spain). 
carried out: for example, if all the spectral pseudo-abscissa values of $J f\left(z_{0}\right)$ are negative, the periodic solutions are asymptotically stable.

The result on the existence of periodic solutions via the averaging method can also be extended to nonsmooth systems (that is, $F$ is merely continuous on $z$ ), like in [9. Theorem IV.13]. In such a case, the condition $\operatorname{det} J f\left(z_{0}\right) \neq 0$ is replaced by the more general assumption that the Brouwer degree of $f$ is non-vanishing in some neighborhood of $z_{0}$. Since nonsmooth systems appear frequently in applications (simple operations as the max-operator or existence of constraints lead inevitably to a loss of differentiability) it is important to study the corresponding stability problem. In 15, it has been assumed that the nonsmooth system (1.1) admits the constant solution $x \equiv z_{0}$ and that $F$ is (Lipschitz on $z$ and) independent of $\varepsilon$. In that case, a study of stability of the constant solution has been carried out, via the global asymptotic stability of the averaged system $y^{\prime}=\varepsilon f(y)$; see [15, Theorem 2]. In [10, Theorem 3.1] it has been proved that the same result holds when $F$ is continuous and degree zero homogeneous. Both approaches make use of Lyapunov functions and converse Lyapunov theorems.

In this work we study the stability of the nontrivial periodic solutions of (1.1) and we show that the assumption that the function $z \mapsto F(s, z, \varepsilon)$ is $C^{1}$ (for all $s \in[0, T]$ and $\varepsilon \in[0,1])$ can be relaxed to the mere strict differentiability of $z \mapsto F(s, z, 0)$ at $z=z_{0}$ for almost all $s \in[0, T]$. In this case we show in particular that $f$ will be differentiable at $z_{0}$ (Proposition 3.1), hence the study of stability of the periodic solution can again be carried out via the eigenvalues (spectral pseudo-abscissa) of the Jacobian matrix of $f$ at $z_{0}$ (Theorem 3.6).

The proof of our main result can be roughly summarized as follows: We study the Poincaré-Andronov operator, $P(\cdot, \varepsilon)$, of the system (1.1). This operator is locally Lipschitz, but not differentiable. Instead of the classical derivative we work with the generalized Jacobian (see [5, Section 2.6] and (3.1) below). We prove that, for $\varepsilon>0$ sufficiently small, all the generalized Jacobian matrices at a fixed point $z_{\varepsilon}$ of $P(\cdot, \varepsilon)$ are close to the Jacobian matrix of $I+\varepsilon f$ at $z_{0}$. This simple fact yields for example the asymptotic stability of the system (1.1) in the case that $J f\left(z_{0}\right)$ is normal with negative pseudo-abscissa values (Theorem 3.6) or the instability in case that $J f\left(z_{0}\right)$ has all its eigenvalues with positive real part (Theorem 3.8).

Let us finally mention, without entering into detail, that our main results can potentially be applied to the study of stability of limit cycles of the nonsmooth system considered in [4]. As an illustration, we study a simplified planar system at the end of Section 3.

\section{NotATION AND PRELIMINARIES}

In this section we fix our notation and present some useful basic results. Throughout this work we shall deal with the system (1.1) and we shall assume that $z_{0} \in \Omega$ is a zero of the function $f$ given in (1.2). Unless otherwise stated, we shall always consider the Euclidean norm $\|\cdot\|$ on $\mathbb{R}^{n}$ and the corresponding operator norm on the space of $n \times n$ matrices. Let us fix a ball $B\left(z_{0}, r_{0}\right) \subset \Omega$ centered at $z_{0}$ with radius $r_{0}>0$. Then for each $z \in B\left(z_{0}, r_{0}\right)$ we denote by $x(\cdot, z, \varepsilon):\left[0, t_{(z, \varepsilon)}\right) \rightarrow \mathbb{R}^{n}$ the solution of (1.1) with initial point $x(0, z, \varepsilon)=z$. Using the local existence and uniqueness theorem (see [7, Section 1.3], for example) we deduce that there exists $\varepsilon_{0} \in(0,1]$ (sufficiently small) such that $t_{(z, \varepsilon)}>T$ for all $z \in B\left(z_{0}, r_{0}\right)$ and all $\varepsilon \in\left[0, \varepsilon_{0}\right]$. 
We define the Poincaré-Andronov operator as follows:

$$
P: B\left(z_{0}, r_{0}\right) \times\left[0, \varepsilon_{0}\right] \rightarrow \mathbb{R}^{n} \quad \text { with } \quad P(z, \varepsilon):=x(T, z, \varepsilon) .
$$

We recall that a solution $x(\cdot, z, \varepsilon)$ of (1.1) satisfying $x(0, z, \varepsilon)=x(T, z, \varepsilon)$ is $T$ periodic. Thus, $z_{\varepsilon}$ is a fixed point of $P(\cdot, \varepsilon)$ if, and only if, $x\left(\cdot, z_{\varepsilon}, \varepsilon\right)$ is a $T$-periodic solution of (1.1).

Definition 2.1 (Asymptotic stability). (i) A fixed point $z^{*}$ of the operator $P(\cdot, \varepsilon)$ is called stable if for each $\eta>0$ there exists $\delta>0$ such that $\left\|z-z^{*}\right\|<\delta$ implies $\left\|P^{n}(z, \varepsilon)-z^{*}\right\|<\eta$ for all $n \geq 0$. A stable fixed point for which there exists $\delta>0$ such that for all $z \in B\left(z^{*}, \delta\right)$

$$
\lim _{n \rightarrow+\infty}\left\|P^{n}(z, \varepsilon)-z^{*}\right\|=0,
$$

is called asymptotically stable.

(ii) A periodic solution $x\left(\cdot, z^{*}, \varepsilon\right)$ of the system (1.1) is called stable (in the sense of Lyapunov) if for each $\eta>0$ there exists $\delta>0$ such that $\left\|z-z^{*}\right\|<\delta$ implies $\left\|x(t, z, \varepsilon)-x\left(t, z^{*}, \varepsilon\right)\right\|<\eta$ for all $t \geq 0$. A stable periodic solution for which there exists $\delta>0$ such that for all $z \in B\left(z^{*}, \delta\right)$

$$
\lim _{t \rightarrow+\infty}\left\|x(t, z, \varepsilon)-x\left(t, z^{*}, \varepsilon\right)\right\|=0
$$

is called asymptotically stable.

The following result shows that the study of asymptotical stability of the periodic solutions of (1.1) can be reduced to the study of the Poincaré-Andronov operator (see [6], for example).

Theorem 2.2 (Periodic solutions vs. Poincaré-Andronov operator). For fixed $\varepsilon>$ $0, z_{\varepsilon}$ is an asymptotically stable fixed point of $P(\cdot, \varepsilon)$ if, and only if, $x\left(\cdot, z_{\varepsilon}, \varepsilon\right)$ is an asymptotically stable periodic solution of (1.1).

In view of this theorem and the above definitions, it is easy to see that if there exists $\delta>0$ and $0<\rho<1$ such that

$$
\left\|P\left(z_{1}, \varepsilon\right)-P\left(z_{2}, \varepsilon\right)\right\| \leq \rho\left\|z_{1}-z_{2}\right\| \text { for all } z_{1}, z_{2} \in B\left(z^{*}, \delta\right),
$$

then the fixed point $z^{*}$ of $P(\cdot, \varepsilon)$ will be asymptotically stable, thus the same holds for the periodic solution $x\left(\cdot, z^{*}, \varepsilon\right)$ of (1.1) as well.

Using the continuous dependence of the solution with respect to initial condition $z \in B\left(z_{0}, r_{0}\right)$ and parameter $\varepsilon \in\left[0, \varepsilon_{0}\right]$ (see [7, Section 1.3], for example), we deduce that there exists a compact set $K$ with $B\left(z_{0}, r_{0}\right) \subset K \subset \Omega$ such that for all $(t, z, \varepsilon) \in[0, T] \times B\left(z_{0}, r_{0}\right) \times\left[0, \varepsilon_{0}\right]$

$$
x(t, z, \varepsilon) \in K .
$$

Let $M>0$ be such that for all $t \in[0, T], x \in K$ and $\varepsilon \in\left[0, \varepsilon_{0}\right]$

$$
\|F(t, x, \varepsilon)\| \leq M \text {. }
$$

Using compactness of $K$ we also deduce the existence of a uniform Lipschitz constant $L>0$ of $F$ such that for all $t \in[0, T]$ and $\varepsilon \in\left[0, \varepsilon_{0}\right]$

$$
\|F(t, x, \varepsilon)-F(t, y, \varepsilon)\| \leq L\|x-y\|, \text { for all } x, y \in K \text {. }
$$

Let us finally mention the equivalent to (1.1) the integral equation for $x(\cdot, z, \varepsilon)$ :

$$
x(t, z, \varepsilon)=z+\varepsilon \int_{0}^{t} F(s, x(s, z, \varepsilon), \varepsilon) d s
$$


for all $t \in[0, T], z \in B\left(z_{0}, r_{0}\right)$ and $\varepsilon \in\left[0, \varepsilon_{0}\right]$. Combining (2.4) with (2.5) and applying the classical Gronwall Lemma, we obtain for each $t \in[0, T]$ and $\varepsilon \in\left[0, \varepsilon_{0}\right]$,

$$
\|x(t, z, \varepsilon)-x(t, w, \varepsilon)\| \leq e^{\varepsilon L t}\|z-w\|, \text { for all } z, w \in B\left(z_{0}, r_{0}\right) .
$$

\section{MAIN RESUltS}

We shall now study the stability of the periodic solutions of (1.1) (or equivalently of (2.5) ). In this work the $C^{1}$ assumption on $F$ is relaxed as follows:

$\left(\mathcal{H}_{1}\right) \quad F(s, \cdot, 0)$ is strictly differentiable at $z_{0}$, for almost all $s \in[0, T]$.

In other words, for almost all $s \in[0, T]$, there exists $A(s):=J F\left(s, z_{0}, 0\right)$ such that

$$
\lim _{z \rightarrow z_{0} u \rightarrow 0}\|u\|^{-1}\|F(s, z+u, 0)-F(s, z, 0)-A(s) u\|=0 .
$$

Note that the existence of the Jacobian $J F$ is no more required for points $(s, z, \varepsilon)$ with either $\varepsilon>0$ or $z \neq z_{0}$. For given (fixed) values of $s \in[0, T]$ and $\varepsilon \in\left[0, \varepsilon_{0}\right]$, we shall consider instead the generalized Jacobian $\partial F(s, z, \varepsilon)$, which is defined as the closed convex envelope of all possible limits of Jacobians of points $\left(s, z_{n}, \varepsilon\right)$ of differentiability of $F(s, \cdot, \varepsilon)$ as $z_{n} \rightarrow z$ ([5, Definition 2.6.1]). An even more general description can be found in [12, Theorem 9.62]. More precisely, if $D_{F}(s, \varepsilon)$ denotes the points of differentiability of $F(s, \cdot, \varepsilon)$, it follows by the Rademacher theorem that $\mathbb{R}^{n} \backslash D_{F}(s, \varepsilon)$ is of Lebesgue measure zero. Then given any null subset $N$ of $\mathbb{R}^{n}$ the following formula holds:

$$
\partial F(s, z, \varepsilon)=\overline{\mathrm{co}}\left\{B_{\varepsilon}=\lim _{z_{n} \rightarrow z} J F\left(s, z_{n}, \varepsilon\right) ; \quad z_{n} \in D_{F}(s, \varepsilon) \backslash N\right\} .
$$

We shall also need to make the following mild assumption, which can be seen as a relaxation of the continuous dependence of the Jacobian $J F$ on the parameter $\varepsilon \in\left[0, \varepsilon_{0}\right]$ in the $C^{1}$ case.

$\left(\mathcal{H}_{2}\right) \quad$ For all $\eta>0$, there exists $\delta>0$ such that for all $z \in B\left(z_{0}, \delta\right)$,

$$
\varepsilon \in[0, \delta] \text { and } s \in[0, T]: \quad \partial F(s, z, \varepsilon) \subset \partial F\left(s, z_{0}, 0\right)+B(0, \eta) \text {. }
$$

Before we proceed, let us observe that $\left(\mathcal{H}_{1}\right)$ has the following useful consequence (the interested reader might want to compare this result with [5, Theorem 7.4.1]):

Proposition 3.1 (Strict differentiability of $f$ ). Under the assumption $\left(\mathcal{H}_{1}\right)$, the function $f$ defined in (1.2) is strictly differentiable at $z_{0}$ with derivative

$$
J f\left(z_{0}\right)=\int_{0}^{T} A(s) d s
$$

Proof. Set $A(s):=J F\left(s, z_{0}, 0\right)$ for all $s \in[0, T]$ where the Jacobian exists, and note that the set $\{A(s): s \in[0, T]$ a.e. $\}$ is bounded by the Lipschitz constant $L$ of $F$. Thus $A=\int_{0}^{T} A(s) d s$ is well-defined. We need to prove the equality

$$
\lim _{z \rightarrow z_{0} u \rightarrow 0}\|u\|^{-1}\|f(z+u)-f(z)-A u\|=0 .
$$


To this end, consider the sequences $z_{n} \rightarrow z_{0}, u_{n} \rightarrow 0$. The conclusion follows using the inequality

$$
\frac{\left\|f\left(z_{n}+u_{n}\right)-f\left(z_{n}\right)-A u_{n}\right\|}{\left\|u_{n}\right\|} \leq \int_{0}^{T} \frac{\left\|F\left(s, z_{n}+u_{n}, 0\right)-F\left(s, z_{n}, 0\right)-A(s) u_{n}\right\|}{\left\|u_{n}\right\|} d s
$$

assumption $\left(\mathcal{H}_{1}\right)$ and the Lebesgue dominated convergence theorem.

Let us introduce for every $\varepsilon \geq 0$ the functions $g_{\varepsilon}, h_{\varepsilon}: B\left(z_{0}, r_{0}\right) \rightarrow \mathbb{R}^{n}$ defined by

$$
g_{\varepsilon}(z)=\int_{0}^{T} F(s, x(s, z, \varepsilon), \varepsilon) d s
$$

and

$$
h_{\varepsilon}(z)=\int_{0}^{T}[F(s, x(s, z, \varepsilon), \varepsilon)-F(s, z, 0)] d s .
$$

Observe that $h_{\varepsilon}(z)=g_{\varepsilon}(z)-f(z)$ and $g_{0}(z)=f(z)$.

Proposition 3.2 (Lipschitz estimation of $h_{\varepsilon}$ around $z_{0}$ ). Under the assumptions $\left(\mathcal{H}_{1}\right)$ and $\left(\mathcal{H}_{2}\right)$, for every $\eta>0$ there exists $\delta>0$ such that for all $\varepsilon \leq \delta$, the function $h_{\varepsilon}$ is Lipschitz on $B\left(z_{0}, \delta\right)$ with a Lipschitz constant at most equal to $\eta$, that is,

$$
\left\|h_{\varepsilon}(w)-h_{\varepsilon}(z)\right\| \leq \eta\|w-z\| \text { for all } w, z \in B\left(z_{0}, \delta\right)
$$

Proof. Set

$$
M_{1}=L T e^{L T} \int_{0}^{T}\|A(s)\| d s \quad \text { and } \quad M_{2}=T\left(1+e^{L T}\right)
$$

and fix any $\eta>0$. Using $\left(\mathcal{H}_{2}\right)$, choose $0<\delta_{1} \leq r_{0}$ such that for all $z \in B\left(z_{0}, \delta_{1}\right)$ all $\varepsilon \in\left(0, \delta_{1}\right)$ and all $s \in[0, T]$ the following relation holds:

$$
\partial F(s, z, \varepsilon) \subset \partial F\left(s, z_{0}, 0\right)+B\left(0, \frac{\eta}{2 M_{2}}\right) .
$$

Let

$$
\delta=\min \left\{\varepsilon_{0}, \frac{\delta_{1}}{2}, \frac{\delta_{1}}{2 M T}, \frac{\eta}{2 M_{1}}\right\}
$$

and fix any $0<\varepsilon \leq \delta$. By (2.5) we deduce that

$$
\|x(s, z, \varepsilon)-z\| \leq \varepsilon \int_{0}^{s}\|F(t, x(t, z, \varepsilon), \varepsilon)\| d t \leq \varepsilon M T \leq \delta_{1} / 2,
$$

thus for every $z \in B\left(z_{0}, \delta_{1} / 2\right)$ and $s \in[0, T]$ we get

$$
x(s, z, \varepsilon) \in B\left(z_{0}, \delta_{1}\right) .
$$

Now let $z, w \in B\left(z_{0}, \delta_{1} / 2\right)$, with $z \neq w$. To simplify notation we shall write in the sequel $x$ instead of $x(s, z, \varepsilon)$ and $y$ instead of $x(s, w, \varepsilon)$. Applying [5, Proposition 2.6.5] (generalized mean value theorem) we obtain $\xi^{*}(s) \in \operatorname{co} \partial F(s,[x, y], \varepsilon)$, $\rho^{*}(s) \in \operatorname{co} \partial F(s,[z, w], 0)$ such that

$$
F(s, y, \varepsilon)-F(s, x, \varepsilon)=\left\langle\xi^{*}(s), y-x\right\rangle
$$

and

$$
F(s, w, 0)-F(s, z, 0)=\left\langle\rho^{*}(s), w-z\right\rangle
$$


Since $z, w, x, y \in B\left(z_{0}, \delta_{1}\right)$, using (3.5) and the convexity of the norm we deduce that for almost all $s \in[0, T]$,

$$
\xi^{*}(s), \rho^{*}(s) \in B\left(A(s), \frac{\eta}{2 M_{2}}\right) .
$$

Note that

$$
\left\|h_{\varepsilon}(w)-h_{\varepsilon}(z)\right\| \leq \int_{0}^{T}\|F(s, y, \varepsilon)-F(s, x, \varepsilon)-(F(s, w, 0)-F(s, z, 0))\| d s ;
$$

thus in view of (3.8), (3.9) and (3.10)

$\left\|h_{\varepsilon}(w)-h_{\varepsilon}(z)\right\| \leq \int_{0}^{T}\|\langle A(s), y-x-(w-z)\rangle\| d s+\frac{\eta}{2 M_{2}} \int_{0}^{T}(\|y-x\|+\|w-z\|) d s$.

In view of (2.6), we get $\|y-x\| \leq e^{\varepsilon L s}\|z-w\|$, thus since $\varepsilon \leq 1$ and $s \leq T$ it follows from (3.4) that

$$
\frac{\eta}{2 M_{2}} \int_{0}^{T}(\|y-x\|+\|w-z\|) d s \leq(\eta / 2)\|w-z\| .
$$

On the other hand, since

$$
\begin{aligned}
\int_{0}^{s}\|F(t, y, \varepsilon)-F(t, x, \varepsilon)\| d t & \leq L \int_{0}^{s}\|y-x\| d t \\
& \leq L\|w-z\| \int_{0}^{s} e^{\varepsilon L t} d t \leq L T e^{L T}\|w-z\|,
\end{aligned}
$$

it follows from (2.5) and (3.6) that

$$
\begin{aligned}
\int_{0}^{T} \|\langle A(s), y-x-(w-z)\rangle|| d s & \leq \varepsilon\left(L T e^{L T} \int_{0}^{T}|A(s)| d s\right)\|w-z\| \\
& \leq(\eta / 2)\|w-z\| .
\end{aligned}
$$

Combining this last relation with (3.11) and (3.12), the assertion follows.

Using Proposition 3.2 we obtain the following result.

Proposition 3.3 (Persistence of generalized Jacobians around $z_{0}$ ). Under the assumptions $\left(\mathcal{H}_{1}\right)$ and $\left(\mathcal{H}_{2}\right)$, for every $\eta>0$ there exists $\delta>0$ such that for all $\varepsilon \leq \delta$, all $z \in B\left(z_{0}, \delta\right)$ and all generalized Jacobians $\Gamma_{\varepsilon} \in \partial g_{\varepsilon}(z)$ we have

$$
\left\|\Gamma_{\varepsilon}-A\right\| \leq \eta,
$$

where $A=J f\left(z_{0}\right)$ (cf. Proposition 3.1).

Proof. Note that $g_{\varepsilon}(z)=f(z)+h_{\varepsilon}(z)$ and that all three functions are locally Lipschitz, thus differentiable almost everywhere. Let us denote by $D_{f}$ (respectively, $D_{g}, D_{h}$ ) the points of differentiability of $f$ (respectively, $g_{\varepsilon}, h_{\varepsilon}$ ), and $N=\mathbb{R}^{n} \backslash$ $\left(D_{f} \cap D_{h}\right)$. Note that $N$ is a Lebesgue null set, thus the generalized Jacobian of $g_{\varepsilon}$ is given by the formula:

$$
\partial g_{\varepsilon}(z)=\overline{\mathrm{co}}\left\{B_{\varepsilon}=\lim _{z_{n} \rightarrow z}\left(J f\left(z_{n}\right)+J h_{\varepsilon}\left(z_{n}\right)\right) ; z_{n} \in D_{f} \cap D_{h}\right\} .
$$

Fix $\eta>0$ and let $B_{\varepsilon}$ be defined as in the above formula. Then applying Proposition 3.2 for $\eta_{1}=\eta / 2$ we obtain $\delta_{1}>0$ such that for all $0<\varepsilon \leq \delta_{1}$, the function $h_{\varepsilon}(\cdot)$ is Lipschitz with constant at most $\eta / 2$ on $B\left(z_{0}, \delta_{1}\right)$. This yields $\left\|J h_{\varepsilon}(w)\right\| \leq \eta / 2$ 
for all $\varepsilon \leq \delta_{1}$ and all $w \in D_{h} \cap B\left(z_{0}, \delta_{1}\right)$. Since $\partial f$ is upper semicontinuous and $\partial f\left(z_{0}\right)=\{A\}$, there exists $\delta_{2}>0$ such that $\partial f(w) \subset B(A, \eta / 4)$ for all $w \in B\left(z_{0}, \delta_{2}\right)$. Let $\delta=\min \left\{\delta_{1}, \delta_{2}\right\}$ and fix $z \in B\left(z_{0}, \delta / 2\right)$. Let $n_{1}>0$ be such that for all $n \geq n_{1}$ we have $\left\|B_{\varepsilon}-\left(J f\left(z_{n}\right)+J h_{\varepsilon}\left(z_{n}\right)\right)\right\| \leq \eta / 4$ and $z_{n} \in B(z, \delta / 2)$. In particular, since $z_{n} \in B\left(z_{0}, \delta_{1}\right)$ for $n \geq n_{1}$ we obtain

$$
\left\|J h_{\varepsilon}\left(z_{n}\right)\right\| \leq \eta / 2 \quad \text { and } \quad\left\|J f\left(z_{n}\right)-A\right\| \leq \eta / 4 .
$$

This yields

$$
\left\|B_{\varepsilon}-A\right\| \leq\left\|B_{\varepsilon}-\left(J f\left(z_{n}\right)+J h_{\varepsilon}\left(z_{n}\right)\right)\right\|+\left\|J f\left(z_{n}\right)-A\right\|+\left\|J h_{\varepsilon}\left(z_{n}\right)\right\| \leq \eta .
$$

Thus for all $\varepsilon \leq \delta$ and all $z \in B\left(z_{0}, \delta\right)$ we get $\left\|B_{\varepsilon}-A\right\| \leq \eta$, and the result follows from the convexity of the ball $B(A, \eta)$.

We further denote by $\operatorname{spec}(\Gamma)$ the (complex) eigenvalues of the matrix $\Gamma$. Using the continuity of the spectral mapping we directly obtain the following corollary.

Corollary 3.4 (Spectral stability of the generalized Jacobians). Fix any $\eta>0$ and assume $\operatorname{spec}(A)=\left\{\lambda_{1}, \ldots, \lambda_{k}\right\}$ for $A=J f\left(z_{0}\right)$. Then there exists $\delta_{0}>0$ such that for all $\varepsilon \in\left[0, \delta_{0}\right], z \in B\left(z_{0}, \delta_{0}\right)$ and $\Gamma_{\varepsilon} \in \partial g_{\varepsilon}(z)$, and for all $i \in\{1, \ldots, k\}$,

$$
\operatorname{spec}\left(\Gamma_{\varepsilon}\right) \bigcap B\left(\lambda_{i}, \eta\right) \neq \emptyset \quad \text { and } \quad \operatorname{spec}\left(\Gamma_{\varepsilon}\right) \subset \bigcup_{i=1}^{k} B\left(\lambda_{i}, \eta\right) \text {. }
$$

Let us discuss an application of the above result. Let us consider the set of zeros of the mapping $(z, \varepsilon) \mapsto g_{\varepsilon}(z)$, or equivalently, the set of fixed points of the Poincaré-Andronov operator $P$ defined in (2.1), that is,

$$
\begin{aligned}
Z & =\left\{\left(z_{\varepsilon}, \varepsilon\right) \in B\left(z_{0}, \delta\right) \times\left[0, \varepsilon_{0}\right]: g_{\varepsilon}\left(z_{\varepsilon}\right)=0\right\} \\
& =\left\{\left(z_{\varepsilon}, \varepsilon\right) \in B\left(z_{0}, \delta\right) \times\left[0, \varepsilon_{0}\right]: P\left(z_{\varepsilon}, \varepsilon\right)=z_{\varepsilon}\right\} .
\end{aligned}
$$

In the $C^{1}$ case, (existence and) asymptotic stability of the periodic solutions is ensured if the Jacobian of $f$ has negative spectral pseudo-abscissa values. In fact, the $C^{1}$ assumption can be replaced by the assumption that for each $\varepsilon>0$ the Poincaré-Andronov operator $P(\cdot, \varepsilon)$ has a fixed point $z_{\varepsilon}$ and is differentiable there. Indeed, in such a case, shrinking $\varepsilon$ if necessary, we deduce from the above corollary that the matrix $\operatorname{JP}\left(z_{\varepsilon}, \varepsilon\right)$ will have all its eigenvalues inside the unit disk of the complex plain. Let us extend this result to the nonsmooth case.

Theorem 3.5 (Existence and asymptotic stability of periodic solutions - I). Let $z_{0} \in f^{-1}(0)(f$ is defined in (1.2) $)$ and let $\left(\mathcal{H}_{1}\right),\left(\mathcal{H}_{2}\right)$ hold true. Assume $\operatorname{det}\left(J f\left(z_{0}\right)\right) \neq 0$ and that for some equivalent norm $|\cdot|$ of $\mathbb{R}^{n}$ (that does not depend on $\varepsilon)$ :

$$
|(z+\varepsilon f(z))-(w+\varepsilon f(w))| \leq(1-\omega \varepsilon)|z-w|
$$

for all $z, w$ around $z_{0}$, where $\omega>0$. Then for every $\varepsilon>0$ sufficiently small the system (1.1) has a unique and asymptotically stable periodic solution near $z_{0}$ with initial value $z_{\varepsilon}$ satisfying $\lim _{\varepsilon \rightarrow 0} z_{\varepsilon}=z_{0}$.

Proof. Let us observe that the assumption $z_{0} \in f^{-1}(0)$ simply means that $\left(z_{0}, 0\right) \in$ $Z$, while the fact that $\operatorname{det} J f\left(z_{0}\right) \neq 0$ yields that $z_{0}$ is isolated in $f^{-1}(0)$ and its topological index with respect to $f$ does not vanish. Thus from the theory of 
topological degree we deduce (see also [3]) that for all $\varepsilon>0$ small enough there exists $z_{\varepsilon}$ with $\left(z_{\varepsilon}, \varepsilon\right) \in Z$ and $\lim _{\varepsilon \rightarrow 0} z_{\varepsilon}=z_{0}$.

Fix $\varepsilon>0$ and apply Proposition 3.2 (which remains true for the equivalent norm $|\cdot|)$ for $\eta \leq \omega / 2$. We conclude that the $|\cdot|$-norm of any generalized Jacobian $\partial h_{\varepsilon}(z)$ around $z_{0}$ is bounded by $\omega / 2$. Since $P(\cdot, \varepsilon)=I+\varepsilon f+\varepsilon h_{\varepsilon}$, it follows easily that

$$
|P(z, \varepsilon)-P(w, \varepsilon)| \leq(1-(\omega / 2) \varepsilon)|z-w|
$$

which for $\varepsilon>0$ sufficiently small guarantees uniqueness and asymptotic stability of the fixed point $z_{\varepsilon}$.

In what follows, we denote by $r(\Gamma)$ the spectral radius of the matrix $\Gamma$, that is,

$$
r(\Gamma)=\max \{|\lambda|: \lambda \in \operatorname{spec}(\Gamma)\} .
$$

Theorem 3.6 (Existence and asymptotic stability of periodic solutions - II). Let $z_{0} \in f^{-1}(0)$ and assume $\left(\mathcal{H}_{1}\right)$ and $\left(\mathcal{H}_{2}\right)$. Then the conclusion of Theorem 3.5 remains true provided that the matrix $A=J f\left(z_{0}\right)$ is normal (thus diagonalizable in $\mathbb{C})$ and

$$
\alpha=\max \{\operatorname{Re}(\lambda): \lambda \in \operatorname{spec}(A)\}<0 .
$$

Proof. Since the eigenvalues of the matrix $I+\varepsilon A$ are of the form $1+\varepsilon \lambda$ for $\lambda \in$ $\operatorname{spec}(A)$, it follows that

$$
r(I+\varepsilon A)<\sqrt{1-2|\alpha| \varepsilon+\|\left. A\right|^{2} \varepsilon^{2}} .
$$

On the other hand, the assumption on $A$ implies that the matrix $I+\varepsilon A$ has $n$ linearly independent orthonormal eigenvectors in $\mathbb{C}^{n}$, which guarantees that

$$
\max \left\{\|(I+\varepsilon A) z\|_{\mathbb{C}}: z \in \mathbb{C}^{n},\|z\|=1\right\}=r(I+\varepsilon A),
$$

thus

$$
\|I+\varepsilon A\|=r(I+\varepsilon A) .
$$

Let $\delta_{0} \leq|\alpha| /|| A||^{2}$ and shrink it further if necessary to ensure that for all $\varepsilon \in\left(0, \delta_{0}\right]$ we have the estimate:

$$
\|I+\varepsilon A\|<1-\left(|\alpha|-2^{-1}\|A\|^{2} \varepsilon\right) \varepsilon<1-(|\alpha| / 2) \varepsilon .
$$

From the strict differentiability of $f$ at $z_{0}$ we deduce that, for all $z, w$ around $z_{0}$,

$$
\|f(z)-f(w)-A(z-w)\| \leq(|\alpha| / 4)\|z-w\| .
$$

Then

$$
\begin{aligned}
\|(z+\varepsilon f(z))-(w+\varepsilon f(w))\| & \leq \varepsilon\|f(z)-f(w)-A(z-w)\|+\|I+\varepsilon A\|\|z-w\| \\
& \leq(1-(|\alpha| / 4) \varepsilon)\|z-w\| .
\end{aligned}
$$

Hence (3.14) is valid around $z_{0}$, thus the conclusion follows from Theorem 3.5.

Remark 3.7. The normality assumption in the above theorem has been used to ensure that $\|I+\varepsilon A\|=r(I+\varepsilon A)$ for all $\varepsilon>0$. In fact, for any matrix $A$ satisfying (3.15) and any $r(I+\varepsilon A)<\rho<1$, one can always define an equivalent norm $|\cdot|$ in $\mathbb{R}^{n}$ for which the corresponding operator norm satisfies $|I+\varepsilon A|<\rho$. However, this norm depends on $\varepsilon$ and eventually becomes large as $\varepsilon \rightarrow 0$.

The following result concerns instability of the periodic solutions. 
Theorem 3.8 (Unstable periodic solutions). Let $z_{0}$ be a zero of the function $f$ defined in (1.2) and assume that $\left(\mathcal{H}_{1}\right),\left(\mathcal{H}_{2}\right)$ hold true and that the matrix $A=$ $J f\left(z_{0}\right)$ is normal and

$$
\beta=\min \{\operatorname{Re}(\lambda): \lambda \in \operatorname{spec}(A)\}>0 .
$$

Then for all $\varepsilon>0$ sufficiently small, (1.1) has an unstable periodic solution with initial value near $z_{0}$.

Proof. The existence of (at least one) periodic solution with initial point near $z_{0}$ follows in the same way as in Theorem 3.5. Let us apply Corollary 3.4 for $\eta \leq \beta / 2$. Then there exists $\delta_{0}>0$ such that for all $\varepsilon \in\left(0, \delta_{0}\right]$ and $z \in B\left(z_{0}, \delta_{0}\right)$, every generalized Jacobian matrix $\Gamma_{\varepsilon} \in \partial g_{\varepsilon}(z)$ satisfies $\left\|A-\Gamma_{\varepsilon}\right\|<\beta / 2$. It follows that

$$
\min _{\|x\|=1}\left\{\left\|\left(I+\varepsilon \Gamma_{\varepsilon}\right)(x)\right\|: x \in \mathbb{R}^{n}\right\} \geq \min _{\|z\|=1}\left\{\left\|\left(I+\varepsilon \Gamma_{\varepsilon}\right)(z)\right\|: z \in \mathbb{C}^{n}\right\} \geq 1+\varepsilon \frac{\beta}{2},
$$

which guarantees the invertibility of the mapping $P(\cdot, \varepsilon)$ around any $z \in B\left(z_{0}, \delta_{0}\right)$; see [5. Section 7.1] for details. Moreover, the inverse function $Q(\cdot, \varepsilon)$ is Lipschitz with constant at most $(1+\varepsilon \beta / 2)^{-1}$. Thus, if $z_{\varepsilon} \in B\left(z_{0}, \delta_{0}\right)$ is the initial point of a periodic solution of (1.1), then for every $z \in B\left(z_{0}, \delta_{0}\right)$ we have

$$
\left\|z-z_{\varepsilon}\right\|=\left\|Q(P(z, \varepsilon), \varepsilon)-z_{\varepsilon}\right\| \leq\left(1+\varepsilon \frac{\beta}{2}\right)^{-1}\left\|P(z, \varepsilon)-z_{\varepsilon}\right\| .
$$

This shows that $z_{\varepsilon}$ is a repelling fixed point of $P(\cdot, \varepsilon)$, and the conclusion follows.

Let us give an application to the existence and stability of limit cycles of a (nonsmooth) planar system, which is studied by essentially the same technique, after transformation to polar coordinates.

Example. Consider the following planar system:

$$
\begin{aligned}
& \dot{x_{1}}=-x_{2}+\varepsilon x_{1}\left(1-2 \varphi\left(x_{1}^{2}+x_{2}^{2}\right)\right), \\
& \dot{x_{2}}=x_{1},
\end{aligned}
$$

where $\varepsilon>0$ is a small parameter and $\varepsilon: \mathbb{R}_{+} \rightarrow \mathbb{R}$ is defined as follows:

$$
\varphi(x)= \begin{cases}x, & \text { for } x \in[0,1] \\ 1, & \text { for } x \in(1, \infty) .\end{cases}
$$

Passing to polar coordinates $x_{1}=r \cos \theta, x_{2}=r \sin \theta$, differentiating the relations $r^{2}=x_{1}^{2}+x_{2}^{2}$ and $\theta=\arctan \left(x_{2} / x_{1}\right)$ and eliminating the parameter $t$ we get from (3.17) a one-dimensional equation of the form (1.1), that is,

$$
\dot{r}(\theta)=\varepsilon F(\theta, r, \varepsilon),
$$

where

$$
F(\theta, r, \varepsilon)=\frac{r \cos ^{2} \theta\left(1-2 \varphi\left(r^{2}\right)\right)}{1-\varepsilon 2^{-1} \sin 2 \theta\left(1-2 \varphi\left(r^{2}\right)\right)} .
$$

Then $F: \mathbb{R} \times(0, \infty) \times[0,1] \rightarrow \mathbb{R}$ is continuous, $2 \pi$-periodic with respect to $\theta$, and locally Lipschitz with respect to $r$. Moreover it satisfies $\left(\mathcal{H}_{1}\right)$ and $\left(\mathcal{H}_{2}\right)$ (note that $F(\theta, \cdot, 0)$ is strictly differentiable at every $r>0$ provided $r \neq 1$. Then (1.2) gives

$$
f(r)=\int_{0}^{2 \pi} F(\theta, r, 0) d \theta=\int_{0}^{2 \pi} r \cos ^{2} \theta\left(1-2 \varphi\left(r^{2}\right)\right) d r=\pi r\left(1-\varphi\left(r^{2}\right)\right) .
$$


It is easily seen that $f$ has a unique zero at $r_{0} \in(0, \infty)$ and $f^{\prime}\left(r_{0}\right)<0$ (cf. Proposition 3.1). We conclude that (3.17) has a unique limit cycle that is asymptotically stable.

Let us conclude with the following remark. Nonsmoothness appears naturally in most concrete problems, and leads to the development of the so-called nonsmooth analysis (see for example [1] and references therein, as well as the classical textbooks [5], 12 and [1]). Here, instead of the classical $C^{1}$-assumption, $F$ is assumed to be strictly differentiable only at points of the form $\left(s, z_{0}, 0\right)$, which enlarges the domain of applicability of the theory: in fact, nonsmoothness seldom occurs in a random manner, but instead it is often well-structured: this is the case for instance in problems involving semialgebraic or subanalytic structures; see 2 for example.

\section{ACKNOWLEDGMENTS}

This work has been realized during a visit of the first author at the CRM and the Autonomous University of Barcelona. The first author wishes to thank her hosts for hospitality.

\section{REFERENCES}

[1] D. Aussel, A. Daniilidis \& L. Thibault, Subsmooth sets: functional characterizations and related concepts, Trans. Amer. Math. Soc. 357 (2005), 1275-1301. MR2115366

[2] J. Bolte, A. Daniilidis \& A. Lewis, The Eojasiewicz inequality for nonsmooth subanalytic functions with applications to subgradient dynamical systems, SIAM J. Optim. 17 (2006), 1205-1223. MR2274510

[3] A. Buică \& J. Llibre, Averaging methods for finding periodic orbits via Brouwer degree, Bull. Sci. Math. 128 (2004), 7-22. MR2033097(2004j:34102)

[4] A. Buică \& J. Llibre, Bifurcation of limit cycles from a 4-dimensional center in control systems, International Journal of Bifurcation and Chaos 15 (2005), 2653-2662. MR 2174572 (2006e:34070)

[5] F. Clarke, Optimization and nonsmooth analysis, A Wiley-Interscience Publication. John Wiley \& Sons, Inc., New York, 1983. MR709590 (85m:49002)

[6] J. Guckenheimer \& P. Holmes, Nonlinear oscillations, dynamical systems, and bifurcation of vector fields, Springer-Verlag New York, Heidelberg, Berlin, 1983. MR.709768 (85f:58002)

[7] J. Hale, Ordinary Differential Equations, Robert E. Krieger Publishing Co., 1980. MR587488 (82e:34001)

[8] Y. Hartono \& A.H.P. van der Burgh, Higher-order averaging: periodic solutions, linear systems and an application, Nonlinear Analysis 52 (2003), 1727-1744. MR.1956172 (2003m:34105)

[9] J. Mawhin, Topological degree methods in nonlinear boundary value problems, CBMS Regional Conference Series in Mathematics 40, AMS, Providence, R.I., 1979. MR525202 (80c:47055)

[10] R.T. M'Closkey, An averaging theorem for time-periodic degree zero homogeneous differential equations, Systems Control Lett. 32 (1999), 179-183. MR.1492438 (99b:34089)

[11] B. Mordukhovich, Variational analysis and generalized differentiation. (Vol. I \& II) Grundlehren der Mathematischen Wissenschaften 331, Springer-Verlag, Berlin, 2006.

[12] T. Rockafellar \& R. Wets, Variational analysis, Grundlehren der Mathematischen Wissenschaften 317, Springer-Verlag, Berlin, 1998. MR1491362 (98m:49001)

[13] J. Sanders \& F. Verhulst, Averaging methods in nonlinear dynamical systems, Appl. Math. Sci. 59, Springer, 1985. MR810620 (87d:34065)

[14] S. Schecter, Stable manifolds in the method of averaging, Trans. Amer. Math. Soc. 308 (1988), 159-176. MR946437 (89i:34061)

[15] A.R. Teel, J. Peuteman \& D. Aeyels, Semi-global practical asymptotic stability and averaging, Systems Control Lett. 37 (1999), 329-334. MR.1753260 (2001b:34098) 
Department of Applied Mathematics, Babeş-Bolyai University, Cluj-Napoca 400084, ROMANIA

E-mail address: abuica@math.ubbcluj.ro

Departament de Matemàtiques, Universitat Autònoma de Barcelona, Bellaterra 08193, Spain

E-mail address: arisd@mat.uab.es 